\title{
Principle of relativity and the "hidden" symmetry of matter motion
}

\section{Zhotikov V.G.}

Moscow Institute of Physics and Technology, Dolgoprudny, Moscow Region, Russian Federation;

E-mail: Zhotikov<zhotikov@yandex.ru>;

More than 100 years have passed since the appearance of the relativity theory (RT) on the "physical sky". It is hardly necessary to doubt that RT has contributed significant progress in understanding the nature of space and time. It seems appropriate to bring at least some preliminary results of its impact on modern physical picture of the world. How the dreams and desires of the classics of relativism of the first decade of the last century correspond to the current state of affairs in this area? What was confirmed from the predicted by them, and what should be send to the "baggage" of the history of physical science? Over the past period, physical science has accumulated a sufficient number of questions to the grounds (postulates) of RT. In this paper, we give a brief analysis of the current state of affairs in this area. The focus is on the following: Why Einstein was unable to bring the idea of the complete relativity (i.e. the independence of the laws of nature from the state of motion of observers) to its logical conclusion?

Keywords: Principle of relativity, gauge transformation, conservation laws.

DOI: $10.18698 / 2309-7604-2015-1-585-598$

\section{Introduction}

Paradigm, formulated based on the special theory of relativity (STR) completely dominated for the last 100 years in the physical science. However, during the same time a lot of issues were accumulated in relation to the mathematical basis of the two theories of relativity: both special and general. Moreover, there is hardly any need to prove to anyone common notion that the success of any physical theory is in the perfection of the mathematical apparatus.

We limit ourselves so far by only a few topics, which certainly useful for understanding the essence, realizing, that a full and reasoned analysis of both insights and disappointments that occurred during more than a century long, is clearly beyond the scope of any reasonable volume of a journal article.

The purpose of this paper is to look at the principle of relativity in terms of the laws of symmetry and consequent conservation laws (see the theorem of Noether and its subsequent generalization). The laws of symmetry are plays a major role in establishing the laws of nature (laws of motion and conservation). One of the simplest examples of conservation laws associated with uniform rectilinear motion. It is believed that the laws of physics does not change in uniform rectilinear motion. This statement received the name of the principle of relativity. 
Einstein attempted to extend this principle to any kinds of movements, including accelerated ones. However, he failed to achieve the desired result. This circumstance drew attention of V.A. Fock [1] even in the middle of the last century.

Perfect solution (from the mathematical point of view), of the problem of spreading the principle of relativity to accelerated motion dictates the necessity of uniting coordinate space $R^{4}(x)$, and the momentum space $R^{4}(p)$ in a common geometric structure. These spaces are dual (mutually complementary) to each other, although they belong to two different classes of measurements. Hereafter, we will use the following notation: $x=(t, \overrightarrow{\boldsymbol{r}})$, where $\overrightarrow{\boldsymbol{r}}$ is a 3 -vector of coordinates and $p=(E, \overrightarrow{\boldsymbol{p}}), \overrightarrow{\boldsymbol{p}}$ is a 3 - vector of momentum ${ }^{5}$.

For quite a long time it was believed that $R^{4}(x)$ and $R^{4}(p)$ can be connected by means of Fourier transforms. However, this proved to be not so. These spaces can be perfectly described mathematically in a unified manner through proper selection of the geometric space that could serve as a model to describe the dynamics of particles and fields.

Has the Murkowski space $M^{4}$, i.e. mathematical model of STR, required properties? "The point" in the space $M^{4}$ is something called "elementary event" of which properties we cannot say anything. In other words, $M^{4}$ represents a 4-dimensional world of zero-dimensional events, each of which must meet a certain spatial point taken at one (certain) time moment. This space does not have the ability to give required mathematically perfect properties to its "points" zerodimensional 4-D objects.

The action integral must be invariant under translations in momentum space for the geometric space, which is in fact could satisfy the principle of relativity, for any motion, including the accelerated motion. We have previously shown [2] that the essential requirements are satisfied by the geometric space, which could mathematically correct combine both of the above geometric space. The structure of the desired area must be endowed with the properties of projective space, because only the projective geometrical space is endowed with all the required properties.

All of the above provides an elegant and mathematically perfect solution to the problem of origin of inertial forces in Nature. In addition, along this path we come to a new (dynamic) interpretation of the effects previously attributed to the consequences of the Lorentz transformations. Finally, an important result of our study is "closing" of the many paradoxes of the SRT, pursuing this theory from the very beginning of its birth.

\footnotetext{
${ }^{5}$ Here, and then we use the system of units: $c=1, h=1$.
} 


\section{On the myth of "a single space-time" according to Minkowski}

Recall renowned Minkowski report made in 21 September 1908 at the 80th meeting of German naturalists and physicians in Cologne [3]:

"M. G.! The views on space and time, which I intend to develop in front of you, appeared on the basis of experimental physics. This is their strength. Their tendency is radical. Henceforth the space by itself and time by itself must turn in fiction and only some kind of connection of both should still retain its independence."

The report produced on the audience and on the inheriting generations lasting impression. It took almost a century and what we have today? The answer is obvious: the above is merely a hypothesis, though quite fascinating.

Let us briefly discuss what modern mathematical thought can tell us on the subject. How these Minkowski predictions are justified? In fact, in the beginning of XX century geometric space of a special kind was declared as a mathematical model of space-time of SRT. It was called "Minkowski space".

Science, as we know, is developing according to its own laws peculiar to it. It turned out that in the mid-50s of the last century Russian school of mathematics presented us a gift, which cannot be waived. Essence of the case is described below.

"Points" of Minkowski space $M^{4}$ are the zero-dimensional objects. The name "elementary event" is assigned for each such "point". Each elementary event must correspond to a 3D-spatial point, taken at some particular time. In this regard, we note that the discussion on the topics such as: can the elementary event STR, i.e. "point" (zero-dimensional object) of Minkowski space $M^{4}$ have the energy and momentum? And (or) whether elementary events interact with each other? will continue as long as there will not be understanding of all the consequences of the Keldysh theorem (on this subject, see, e.g., [4]).

The essence of the Keldysh theorem, in the relation to the hypothesis of Minkowski, is as follows: 3D (spatial "point") and 1D ("dot" on the time axis) zero-dimensional objects can be easily mapped $^{6}$ in compact set with the dimension $4 \mathrm{D}$, however, the result of mapping will be represented as a superposition of individual mappings, and not a single 4D zero-dimensional object.

In this regard, we recall the terminology of this issue accepted in modern mathematics. Suppose there are two sets of mappings: $f: X \rightarrow Y$ and $g$ mappings $f$ and $g$. Record: $h=g \circ f$. This record should be read from right to left: if it is written, as $g f, f$ acts earlier, then $g$. The order

\footnotetext{
${ }^{6}$ Continuous mapping is called a zero-dimensional if the inverse image of each point is
} 
of letters in the recording $h=g \circ f$ adopted such as in recording $h=g f$. Obviously, the reverse mapping, i.e. "Splitting" of 4D Minkowski space for $3+1$ or $2+2$, components appear to be not uniquely defined. In other words, we are faced with a very serious problem of micro-causality. Thus, we should wait with the idea of a single 4D-dimensional manifold of "point-events" in the sense of Minkowski as a mathematical model of space-time of SRT. This brings to mind the wellknown parable about the "foreman that joined space and time." After gathering workers, he set the task: to dig a ditch up to the fence exactly to the dinner and at the end of work announced that combined space and time.

Each scientific concept has a limit of the applicability and any system of scientific concepts internally contradictory, paradoxical. Why is that? Because science is developing concept, and the achievement of applicability limits of the old concepts requires the birth of a new concept, which may be accompanied by a profound semantic transformation of the whole scientific world.

Recall, for example, what J. Wheeler [5] wrote in his time: "An object that is the centerpiece of all classical General Theory of Relativity - the four-dimensional space-time geometry - simply does not exist, if you go beyond the classic approach. These arguments suggest that the concept of space-time and time are not the primary concepts in the structure of physical theory.... There is no space-time, there is no time, there is nothing before, nothing after. The question of what will happen in the next moment devoid of meaning."

Let us formulate the brief summary of the above. Minkowski space $M^{4}$ cannot serve as a mathematical model of space-time of our world. It is possible that based not only on their own intuition, but also on common sense, many authors, referring to the 4-dimensional space-time of STR, use for its designation the symbols of the direct product like $M^{4}: R^{3} \times T$. However, the above does not contradict the possibility to write and solve equations of motion of the field particle in 4D form.

\section{The symmetry of motion of matter and momentum space}

As already noted, let us try to look at the principle of relativity in terms of the laws of symmetry. The latter, according to the modern view plays a decisive role in the establishment of conservation laws.

From the point of view on the geometry as a theory of invariants of a group of transformations (Klein ${ }^{7}$ ), the space-time of special relativity (Minkowski space) is a 4-dimensional

\footnotetext{
${ }^{7}$ The point of view of on the geometry as a theory of invariants of a group of transformations was first proposed by Klein in 1872, in his famous "Erlangen program".
} 
real affine (more strictly equiaffine!) space with metric of a particular signature. In other words, the SRT is the theory of invariance of physical laws in isolated systems relatively uniform motions. However, we note that the Lorentz transformations themselves, received hereinafter the title boosts, does not form a group of transformations. This fact tells us that the attribution to Lorentz transformations some special role in all physical science looks, to put it mildly, is clearly unconvincing.

Moreover, no one has yet proven the exclusivity of these transformations in physics, or in other words, the uniqueness of these transformations in the class of equiaffine (linear with single determinant) transformations.

For a long time it was customary to assume that the coordinate and momentum representation, describing the motion of matter, i.e., the coordinate space $R^{4}(x)$ and momentum space $R^{4}(p)$ connected to each other with Fourier transforms. However, this proved to be not so. Their relationship was clearly not trivial. It owes its existence to two important principles of modern physical science: the principle of least action, and the principle of gauge invariance of the laws of Nature (see, e.g., [2] and references to the works cited therein).

If we talk about symmetries that would define uniform rectilinear motions, we share Feynman famous view: "... the symmetry with respect to uniform rectilinear motion leads to the special principle of relativity ..." [6]. In other words, this principle takes place only in the case of uniform linear motion of reference systems. In the case of the accelerated movement, including when it is reduced to a uniform rotation, the latter is no longer true.

What can we say about the symmetries inherent to the accelerated motion? Symmetries of the system determine the types of its interaction with its environment. What new symmetries "come into play" in the transition to the accelerated motion? What is their connection with symmetries of non-accelerated motion? The general theory of relativity (GTR) of Einstein was supposed to give an answer to this question. However, he did not achieve the answer to the desired question on the selected path. Fock [1] had rightly pointed to this fact. Einstein's attempts to extend the principle of relativity to all types of motion of matter proved futile. We find the explanation to this fact in the absence of adequate mathematical apparatus in the hands of the researchers of that time.

In this paper we attempt to present the foundations of a consistent approach of extending the principle of relativity to arbitrary types of motion. At the same time, we share the idea belonging to A. Einstein, that in reality only the sum - "geometry-physics" is given to us and not every term separately [7]. 
We start consideration of this issue with the attempt to understand the meaning of the term "symmetry inherent to accelerated motion." To this end, we will have to deal with an important view of symmetry, somehow rarely considered in the literature, which is called translation in momentum space $R^{4}(p)$

$$
p^{\prime}=p-\pi
$$

where 4-covector $\pi=(E, \boldsymbol{p}) \in R^{4}(p)$ is a function of the point $x=(t, \boldsymbol{r})$ in the coordinate space $R^{4}(x): \pi=\pi(x)$. This type of symmetry is called translation in momentum space or briefly impulse translations.

One of the simplest particular cases of accelerated motion in terms of impulse translation transformations (1) is a motion with constant acceleration. In this case, the 4-vector of impulse translations $\pi(x)$ in the region under consideration is a constant vector. Such movements are called hyperbolic motions. Finally, we note that the vanishing of the translation vector $\pi(x)$, brings us to the case of uniform motion.

In Finsler geometry action is proportional to the length of the world line. The invariance of the equations of motion under the transformations (1) will be achieved if the following coordinate transformations are carried out in a synchronous manner in $R^{4}(x)$

$$
x^{\prime}=\frac{X}{1-\pi(x) \cdot x}
$$

The components of the 4-covector $\pi(x)$ plays as the transformation parameters of (2). The scalar product of $\pi(x) \cdot x$ vectors $\pi(x)$ and $x$ in (2) should be understood as the ratio of the segments determined by these vectors. This ratio has the following properties:

If $\pi(x) \cdot x$, the hyperplane $\pi(x)$ lies between the origin $O$ and the end of the vector $x$.

If $\pi(x) \cdot x=1$, the end of the vector $x$ belongs to the hyperplane $\pi(x)$.

If $0<\pi(x) \cdot x<1$, the end of the vector $x$ lies between the origin $O$ and the hyperplane $\pi(x)$.

If $\pi(x) \cdot x=0$, the vector $x$ lies in the initial hyperplane covector $\pi(x)$.

If $\pi(x) \cdot x<0$, the origin $O$ lies between the end of the vector $x$ and the hyperplane $\pi(x)$.

Using modern mathematical terminology we should speak: conversion (1) of translations in momentum space $R^{4}(p)$ induces the transformations (2) in the coordinate space $R^{4}(x)$. These transformations in projective geometry are called homological transformation or conversions of 
homology. The terms homology and homological conversions were introduced to the geometry by J. Poncelet in 1822. In this regard, we recall that the word homology indicates compliance in translation from the Greek (Greek: Homologia).

An important property of these transformations is the fact that they form a group, which is a subgroup of the central projective transformations. In other words, the homology group and the group of rotations form a group of central-projective transformations.

In this regard, we note the following. In physics, we are dealing with two kinds of transformations: active and passive. Transformations (1), (2), with which we are dealing, provide, on the one hand, real and not kinematic changes in the dimensions (scale) and changes in the tempo of the clock rate (passive transformations), on the other hand changes in the state of motion of the system (active transformations). Thus, the transformations (1) in the momentum space $R^{4}(p)$ induce (i.e., aim, trigger, etc.) transformations (2) in the space $R^{4}(x)$. The reverse assertion is also true.

Transformations (2) of the vectors in $R^{4}(x)$, induce transformations (1) of covariant vectors in $R^{4}(p)$. All this takes place due to the principle of duality inherent to transformations of the group of projective transformations. The consequences of this type of symmetry are impairing effects of the clock rate and alterations of linear scale. In this regard, please note the following.

Let us note that, until recently, these effects are mainly explained as a consequence of the Lorentz transformation. We recall in this regard that the Lorentz transformations link together the coordinates of the same points of Minkowski space defined in two inertial reference frames moving relative to each other with a relative velocity. At the same time (as is often forgotten), the coordinate origins of latter shall at any given time coincide with each other. The latter circumstance leads to a very serious devaluation of experimental basis of SRT of the last century.

One of the important particular cases considered symmetry group are gauge transformations. It is known that the calibration principle along with the variation principle is one of the most important principles of modern physics. The terms "gauge symmetry" and "gauge transformations" were introduced by H.Weyl around 1920. We face in electrodynamics with the famous case of gauge transformations: the electromagnetic field tensor $F_{\mu \nu}$ and Maxwell's equations does not change, if the 4-vector potential of the field $A_{\mu}$ transformed as

$$
A_{\mu}(x) \rightarrow A_{\mu}(x)+\partial_{\mu} f(x), \mu=0,1,2,3
$$


Where $f(x)$ arbitrary scalars function of is coordinates in space $R^{4}(x)$, and $\partial_{\mu} f(x)$, is the 4-gradient vector. More generally, gauge transformations for the dynamics of a system of many particles can be written as follows:

$$
L(q, \dot{q}) \rightarrow L^{\prime}(q, \dot{q})+\partial_{\alpha} F(q)
$$

Here $L(q, \dot{q})$ Lagrange function (Lagrangian) of the generalized coordinates $q \equiv\left\{q^{\alpha}\right\}$ and generalized velocities $\dot{q} \equiv\left\{\dot{q}^{\alpha}\right\}$, where $\mapsto \quad$ the number of degrees of freedom of a dynamic system, and $\partial_{\alpha} F(q)$ gradient vector. Its dimension corresponds to the total number of degrees of freedom $\mapsto$ of studied system. The latter can now be both translational and rotational degrees of freedom. Transformations like (4) in the calculus of variations usually are called Caratheodory transformations, who first applied them to obtain sufficient conditions for functional activities [8].

Transformations (3) or (4) represent a special case of transformations (1), when the vector of impulse translation becomes a gradient of a scalar function. Naturally, the transformations (3) and (4) will induce transformations (2) corresponding to them with all the ensuing consequences.

Thus, the projective geometry finds important applications in physics. First of all, the symmetry inherent for accelerated movements "comes into the play". This, in turn, leads to the new, more general conservation laws inherent to physics of open systems. Simultaneous interdependence of object's (body) state changes are achieved when describing its motion in the momentum representation with the description of its motion in the coordinate representation.

Finally, we note one more nontrivial consequence: the modern mathematical apparatus of projective differential geometry makes it possible to provide a geometric interpretation of gauge transformations in physics.

Recently, there was considerable interest in the group of conformal transformations in the physics literature. Therefore, it is very useful to discuss their connection with the considered group of impulsive translations.

Comparison of conformal geometry with the projective one reveals profound differences between them. F. Klein, however, showed at the beginning of the last century that the fundamental group of conformal geometry is isomorphic to one of the subgroups of the projective group. Circles turn into circles for any transformation of the conformal group. Curves of 2 nd order turns into themselves in transformations of the projective group. In other words, there is no difference between the ellipse (circle), hyperbole and parabola from the point of view of projective geometry. 


\section{Discussion of the results}

Let us now consider the consequences associated with the presence of symmetries inherent to accelerated movements in nature. In this paper, we restrict ourselves to simple manifestation of these motions. This are the motions along flat trajectories, where we incorporate accelerated rectilinear motion.

Differential invariant of Euclidean and pseudo-Euclidean geometry is the square of the differential equiaffine line length (path) $d s^{2}=$ const .

When it comes to accelerated movements, instead of differential invariant $d s^{2}$ inherent to uniform rectilinear motion, which is called equiaffine length element, more general invariant of the motion "comes into play":

$$
d \sigma^{3}=d k \cdot d s^{2}=\text { const }
$$

where $d \kappa$ is differential equiaffine curvature of the trajectory. In the projective differential geometry the cubic root of this value is called the projective length element. Projective length element is null equation for a conic (curves of the second order): $d \sigma \equiv 0$. Moreover, it vanishes at the points of the curve, in which osculating conic is hyper osculating.

The motion of particles (bodies) on such (flat) trajectories (orbits) is a motion by inertia. This condition is satisfied by the curves of the second order: Ellipse (circle), hyperbole and parabola.

In other words, the movement of particles under the condition $d \sigma \equiv 0$ is free movement on the light-like geodesic. This condition is satisfied, for example, with the electrons in the atoms and electrons in superconductors. Now we are able to solve the problem of the interaction of particles with external field and fields generated by themselves and explain the nature of inertial forces.

The following is the answer to the question of what gives us invariant of motion $d \kappa \cdot d s^{2}$ that inherent to the motions at arbitrary flat trajectories. We come to understand the true role of the inertial forces in Nature.

Over the centuries, the problem of the nature of the forces of inertia, excited and continues to agitate the minds of the most deserving people on planet Earth.

We will talk about possible explanation of the physical nature of Newton's forces of inertia. Academician A. Ishlinskiy writes about them in his famous monograph: 
«None of the new terms in the mechanics did not bring in the following so much trouble and misunderstandings, as the Newtonian force of inertia ...» There occur the confusion that continues to this day, ongoing debates are carried out about whether the inertia forces are real or unreal (fictitious) and whether they have the counteraction" (see [13], pp.14 15).

Young readers will be interested to learn that in 1946, academician L. Mandelstam in his last article published subsequently in the Physics-Uspekhi (Advances in Physical Sciences), criticized the author of the russian translation of I. Newton's «Principia», a prominent shipbuilder, academician A.N. Krylov [14]. The essence of the criticism was in the following. Translator allegedly not exactly translated next place in Newton's «Principia», which supposedly was misleading.

«Definition III. Congenital force of matter is its common ability of resistance through which anybody taken separately, since it is left to itself, keeps its state of rest or uniform rectilinear motion. This force is always proportional to the mass, and if it different from the inertial mass, solely by the view on it». «From inertial mass occurs that anybody only with difficulty derived from its rest or motion, so the innate strength could be quite intelligibly called inertial force .... ${ }^{8}$.

In this regard, it should be noted that the given definition in the following led to the concept of Newtonian force of inertia as opposed to another type of inertial forces occurring in the inertial reference systems. However, according to Mandelstam in the cited work: «In fact, of course, there are no inertial forces, either real or fictitious. Apparently, however, the beginning of all disputes is this place in Newton's «Principia»»».

What can we say about this? Above, and even earlier [15], it was shown (and in mathematically perfect form) that the presence of symmetry group inherent to accelerated motions resulting in a corresponding conservation law according to the well-known theorem of E. Noether. In this case, such is the law of conservation of inertia in all physical forms of its content.

The latter fact gives us a base to announce that the discussion on «if epy Newtonian force of inertia real or unreal» is completely exhausted. As for other types of inertial forces (centrifugal and Coriolis ones), they are as real as Newton's forces of inertia. One of our upcoming works will be devoted to their review in terms of our approach with principles of symmetry.

An important consequence of introduction of the concept of projective length element is automatic resolution of numerous paradoxes inherent in the STR that we have received from the Classics of Relativism. We are referring to the well-known Bell paradox [9] and Ehrenfest paradox

${ }^{8}$ A.N. Krylov's translation. 
[10]. Now, mathematically perfect solutions to these paradoxes and others like them are resolved instantly using one of the simplest invariants of accelerated motion of matter (5).

In this regard, we also recall considerations of Einstein on the equivalence between the clocks own time with the length of trajectory of its world line: $\tau \sim \int_{\gamma} d s$, where, as before, $d s^{2}$ is equiaffine differential of path length.

On this occasion, it is useful to draw attention to an important, but little-known fact. A. Sommerfeld, in his note to the Minkowski [3] drew attention to the following: «As Minkowski has mentioned in a conversation with me, the element of proper time $d \tau$ is not a total differential. So when you combine the two world points $O$ and $P$ in two different world lines 1 and 2 , then

$$
\int_{1} d \tau \not \equiv \int_{2} d \tau
$$

If the first world line runs parallel to the axis $t$, then as a result the first transition means standstill in the coordinate system of its founding, it is easy to see that

$$
\int_{1} d \tau=t, \quad \int_{2} d \tau<t
$$

This is the basis of lag of moving clock relative to a stationary one noted by Einstein. This idea is based, as noted by Einstein himself, on (unprovable) assumption that a moving clock does indicate the proper time. This means that they indicate the time that corresponds to the instantaneous state of velocity, which is thought to be fixed. »

We mentioned Sommerfeld considerations not by chance. In fact, Einstein's statement that a moving clock does indicate the proper time, which is proportional to the length of the trajectory, is a hypothesis, which is also unprovable. In essence, this statement is third postulate (principle) of A. Einstein's SRT. In our days, the beautiful name «Principle of locality» was given to it, which should be taken as follows: acceleration does not affect the pace of the clock.

If we follow this hypothesis, then we must admit that at accelerated motion should take place

$$
\tau \sim \int_{\gamma} \sqrt[3]{d k \cdot d s^{2}}
$$


The expression under the integral sign, as we have noted, is called the projective length element. Hence, it is easy to see that described in numerous sources «twin paradox» is now being implemented «exactly the opposite»: the younger one turns out to be the traveler, rather than his lazy twin.

However, that's not all. Our "mages" of SRT has, seemingly, yet another very important "trump card" in their sleeves, of which they are particularly proud. This is the concept of simultaneity. Einstein considered concept of simultaneity in the «point» as self-evident. By this he apparently emphasized that his main interest is focused primarily on the rules of the transformation of the physical laws during the transition from one reference frame to another, which is moving relative to the first one [12].

Yet, in vain. For today, it became already obvious that the thesis of the relativity of simultaneity is substantially yet another of the postulates of our «old SRT». Since this issue requires a separate and serious consideration, we do not dwell on it here and devote the remaining part of this article to the most serious issues we sat in the introduction.

\section{Conclusion}

Fundamental truths are given not easy. It took about 2 thousand years to understand that there are inertial motions from Aristotle to Newton. It took another 300 years to realize that inertial motion can be accelerated from Newton to the present day. Thus, free fall in the gravitational field is the inertial motion in non-inertial reference frame. The motion of test bodies by Kepler's laws just represent accelerated but inertial (state) motions of test bodies motion along inertial trajectories.

Many works have appeared recently, proposing to abandon the Minkowski space as a geometric model of space-time. This topic is very promising, however, requires separate consideration.

Meanwhile, it becomes obvious that Minkowski space as well as an attempt to generalize it to the case of accelerated motions space-time of general relativity theory (Riemann space) cannot be taken as basic geometric models to describe the world in which we live.

Author would like to finish his brief excursion into the base of the theory of relativity with words belonging to one of the great founders of quantum theory V. Heisenberg [16], which perfectly, characterize the essence of learning the truth in the science. 
«Almost every advance in the development of natural sciences achieved at the cost of giving up something preceding; with almost every intellectual step forward it is necessary to sacrifice questions, notions and concepts that have been considered important and significant. Thus, the expansion of knowledge to some extent reduces the claim of scientists to complete understanding of Nature».

\section{References}

1. Fock V.A. (1956). Remarks to the creative biography of A. Einstain. A. Einstain and modern physics. Moscow: GITL.

2. Zhotikov V.G. (2014). On the modern point of view on A. Einstain's principle of general relativity. Journal of Hypercomplex Numbers in Geometry and Physics, № 1 (21), Vol. 11, 21 $-36$.

3. Minkowski H. (1909). Raut und Zeit. Phys. ZS. 10, 104.

4. Keldysh L.V. (1954). On representation zero-dimensional open mapping by the superposition. RAS USSR, № 5, Vol. 98, 719 - 722 .

5. Misner C.W., Thorne K.S., Wheeler J.A. (1973). Gravitation Vol. 3, San Francisco: W.H. Freeman and Company.

6. Feynman R.P. (1997). Six not-so-easy pieces. MA: Helix Books Addison-Wesley.

7. Einstain A. (1923). Jejnshtejn geometrija i opyt [Geometry and the experiment]. Petrograd.

8. Caratheodory C. (1935). Variationsrechenung. Leipzig, Berlin.

9. Bell J.S. (1987). How to teach special relativity. Progress in Scientific Culture, Vol. 1, № 2, 67 $-80$.

10. Ehrenfest P. (1909). Phys. Zeits. 10.

11. Sommerfeld A.A. (1935). Remark to H. Minkowski paper Raut und Zeit. Principle of Relativity. Collection papers founders of relativity. Moscow: ONTI.

12. Einstain A. (1905). Zur. Elektrodynamik bewegter Körper. Ann. d. Phys., Vol. 17.

13. Ishlinskiy A. (1987). Classical mechanics and inertial force's. Moscow: Nauka [Science].

14. Mandelstam L.I. (1946). Wans more about of inertial force's. UFN, Vol. 21. 
Proceedings of International Conference PIRT-2015

15. Zhotikov V.G. (2006). New Symmetries of Space-time and nonlinearity in the Nature. Proceeding of the $5^{\text {th }}$ International Conference Bolyai-Gauss-Lobachevsky (BGL-5), Vol. 5, $76-86$.

16. Heisenberg W. (1953). Philosophical problems of atomic physics. Moscow: I.L., 20 - 33. 